\title{
Size Isn’t Everything - Compositional Variation in Hybrid Organic- Inorganic Lead Halide Perovskites: Kinetically- versus Thermodynamically-controlled Synthesis
}

Jiyu Tian ${ }^{1,2}$, Eli Zysman-Colman ${ }^{2, *}$ and Finlay D. Morrison ${ }^{1, *}$

${ }^{1}$ EaStCHEM School of Chemistry, University of St Andrews, St Andrews, Fife, KY16 9ST, United Kingdom.

${ }^{2}$ Organic Semiconductor Centre, EaStCHEM School of Chemistry, University of St Andrews, St Andrews, Fife, KY16 9ST, United Kingdom.

Key words: organic-inorganic hybrid perovskite, mixed-cation, cation composition, cation size, mechanosynthesis, precipitation synthesis.

\begin{abstract}
The formation and study of a partial solid solution $\mathrm{Az}_{1-x} \mathrm{FA}_{x} \mathrm{PbBr}_{3}$, using 'similar' sized cations azetidinium $\left(\mathrm{Az}^{+}\right)$and formamidinium $\left(\mathrm{FA}^{+}\right)$, was explored via mechanosynthesis and precipitation synthesis. The composition and lattice parameters of samples from both syntheses were analysed by ${ }^{1} \mathrm{H}$ NMR and Rietveld refinement of the powder X-ray diffraction. A clear mismatch in the composition of the perovskite was found between the precipitated samples and the corresponding solutions. Such a mismatch was not observed for samples obtained via mechanosynthesis. The discrepancy suggests products are kinetically-controlled during precipitation, compared to thermodynamically-controlled mechanosynthesis. Furthermore, the cell volume as a function of composition in both $6 \mathrm{H}$ (Az-rich) and 3C (FA-rich) solid solutions suggests that $\mathrm{FA}^{+}$is actually smaller than $\mathrm{Az}^{+}$, contradicting the literature. In the $3 \mathrm{C}$ (Az-poor) solid solutions, the extent of $\mathrm{Az}_{1-x} \mathrm{FA}_{x} \mathrm{PbBr}_{3}$ is unexpectedly smaller than $\mathrm{Az}_{1-x} \mathrm{MA}_{x} \mathrm{PbBr}_{3}$, again in contradiction to the expectation based on the reported cation sizes. These results indicate that other factors, as yet unidentified, must also contribute to the solid solution formation of organicinorganic hybrid perovskites, not simply the relative sizes of the A-site cations.
\end{abstract}




\section{Introduction}

Organic-inorganic hybrid perovskites (OIHPs), which share a general formula $\mathrm{ABX}_{3}$, where the A-cation is typically an organic ammonium group, the B-cation is typically a group 14 cation and most commonly $\mathrm{Pb}(\mathrm{II})$ and the $\mathrm{X}$-anion is a halide. OIHPs have emerged as promising materials for next-generation solar cells, ${ }^{1-3}$ light-emitting diodes, ${ }^{4,5}$ optically-pumped lasers $^{6}$ and photodetectors. ${ }^{7,8}$ The optoelectronic properties of OIHPs are highly dependent on the nature of $\mathrm{B}-\mathrm{X}$ interactions as their orbital interactions determine the formation of valence and conduction bands and band gap. The Goldschmidt tolerance factor, $t$, which relates the relative sizes of the A, $\mathrm{B}$ and $\mathrm{X}$ ionic radii, is a simple metric to ascertain if a stable 3D-perovskite structure is adopted and whether any structural distortion from the cubic aristotype is likely to be present. The most common type of distortion driven by the size of the A-cation is octahedral tilting, which affects the B-X orbital overlap and hence the optical bandgap. ${ }^{9}$ The pioneering work in OIHPs used methylammonium $\left(\mathrm{MA}^{+}\right)$as the A-site cation to produce a cubic 3D-perovskite structure, ${ }^{10}$ with formamidinium $\left(\mathrm{FA}^{+}\right)$also adopting the cubic structure. ${ }^{11}$ The band structure of perovskite materials can be tuned directly by varying the B-cation and the halide (although the choice of suitably large B-cations is rather limited) or indirectly due to structural distortions driven by the A-cation size. As a result, the A- and X-site species are commonly manipulated via solid solution formation in order to tune the optoelectronic properties such as the bandgap, ${ }^{11-13}$ emission energies ${ }^{14,15}$ and photoluminescence lifetime. ${ }^{16,17}$ In addition to structural diversity, ${ }^{18-21}$ other advantages of A-cation doping have been shown to include improved stability ${ }^{22}$ and power conversion efficiency of photovoltaic devices, ${ }^{23}$ and reduced trap states in the fabrication of solar cells. ${ }^{24,25}$ For example, Saliba et al. achieved high efficiency solar cells up to $21.6 \%$ with a mixed A-cation composition - $(\mathrm{Rb}, \mathrm{Cs}, \mathrm{MA}, \mathrm{FA}) \mathrm{PbI}_{3}$, which retained $95 \%$ power conversion efficiency after 500 hours at $85{ }^{\circ} \mathrm{C} .{ }^{26}$ Prochowicz et al. obtained a mixed A-cation solid solution system $\mathrm{MA}_{x} \mathrm{FA}_{1-x} \mathrm{PbI}_{3}$ by mechano-synthesis to stabilise the $\alpha-\mathrm{FAPbI}_{3}$ cubic phase in air, which is a key absorber layer in high-efficient solar cells. ${ }^{22}$

To obtain perovskite solid solutions (e.g., with mixed A-cations), synthetic methods including antisolvent $^{21,27}$ and oversaturation ${ }^{3,9}$ precipitation or mechanosynthesis (grinding) $^{13,28}$ are typically used. Principally, the choice of synthetic method has an impact on the morphology of the perovskite material: precipitation and mechano-synthetic methods have been widely used to obtain 
large single crystals or powders that can then be used for structural and photophysical studies. During precipitation synthesis, however, it is usually assumed that the final (actual) sample composition retains the 'nominal' initial molar ratio of ion sources of the precursor solution. Importantly, this assumes that the kinetics of precipitation are independent of the precursor species and nominal composition. The validity of this assumption is often not checked by compositional analysis but rather 'validated' by the observation of a systematic change in properties. In contrast, during mechanosynthesis, all the precursor materials are retained, and the reaction product is obtained under thermodynamic control in a manner analogous to conventional (mixed salt) high temperature solid-state routes. Under such conditions, the final product(s) must reflect the constituent global starting composition and, therefore, if a single phase (perovskite) product is achieved then this must, perforce, have the nominal starting composition.

Any mismatch between nominal and actual composition may reduce the reliability of the conclusions drawn from subsequent structural and photophysical studies. Examples of nominal and actual composition mismatch in mixed-metal ${ }^{24,29-32}$ and mixed-halide ${ }^{24}$ perovskite systems have been studied using X-ray photoelectron spectroscopy (XPS) and flame atomic absorption spectroscopy (FAAS). These studies demonstrated that the actual amount of $\mathrm{Sn}$ and $\mathrm{Bi}$, incorporated in $\mathrm{FAPb}_{1-x} \mathrm{Sn}_{x} \mathrm{Br}_{3}{ }^{24}$ and Bi-doped $\mathrm{MAPbX}_{3}(\mathrm{X}=\mathrm{Cl}, \mathrm{Br}, \mathrm{I})^{29-31}$ single crystals respectively, was significantly less than the nominal percentages of $\mathrm{Sn}$ and $\mathrm{Bi}$ in the initial respective solution. In another example, the actual $\mathrm{Cl} \%$ in $\mathrm{FAPbBr}_{3-x} \mathrm{Cl}_{x}{ }^{24}$ crystals was found to be larger than the nominal value for $\mathrm{Cl}$-poor target compositions $(<50 \% \mathrm{Cl})$ but smaller for Cl-rich reactions. Similar instances were also found for A-cation substitutions. ${ }^{15,33}$ For instance, Ioannis et al. ${ }^{15}$ demonstrated by NMR analysis that the actual ethylenediammonium (en) incorporated in $\left(\mathrm{MA}_{1-x} \mathrm{en}_{x}\right) \mathrm{PbI}_{3}$ was less than the nominal composition. To account for such mismatches, several explanations ${ }^{29,31,34}$ have been proposed including the difference between valence (and hence induced compensating defects) and ionic radii, and surface effects (especially for XPS analysis) for mixed metal (B-site) solid solutions. Such explanations for mixed halide and mixed A-cation systems are absent as the composition with respect to halide and organic A-cation content is overlooked in most studies. Most compositional studies on nominal and actual compositions of solid solutions with substitutions at $\mathrm{A}, \mathrm{B}$, and $\mathrm{X}$ sites are carried out on samples prepared by precipitation methods and in systems that can support a complete solid solution due to isostructural end-members. Beyond simple observation of lattice parameter variations (Vegard's law), the 
paucity of structural contrast makes it challenging to determine if the system sustains solid solution formation or has phase separation. Considering the compositional consistency of mechanosynthesis, the formation of $6 \mathrm{H}$ and $3 \mathrm{C}$ lead bromide perovskite with mixed organic $\mathrm{A}$ cations is here explored to study the relation between the nominal and actual composition using both NMR and PXRD analyses.

The previously reported solid solution study ${ }^{21}$ of $\mathrm{Az}_{1-x} \mathrm{MA}_{x} \mathrm{PbBr}_{3}$, prepared by antisolvent precipitation, indicated partial solid solutions of the hexagonal $(6 \mathrm{H}) \mathrm{AzPbBr}_{3}$ and cubic (3C) $\mathrm{MAPbBr}_{3}$ parent perovskite structures with an intermediate $6 \mathrm{H}-3 \mathrm{C}$ two phase region for $0.3 \leq x \leq$ 0.8. In this system, there is a large difference in cation size between Az and MA. In the current study, the impact of using similar size cations Az and FA $\left(r_{\mathrm{Az}}=250 \mathrm{pm}, r_{\mathrm{FA}}=253 \mathrm{pm}, r_{\mathrm{MA}}=217\right.$ $\mathrm{pm})^{35}$ in the solid solution $\mathrm{Az}_{1-x} \mathrm{FA}_{x} \mathrm{PbBr}_{3}$ was explored. The hypothesis is that a wider range of solid solutions should exist for this system given the similar cation size. The extent of solid solution formation using both mechanosynthesis and precipitation methods was investigated and compared with two related systems: $\mathrm{Az}_{1-x} \mathrm{MA}_{x} \mathrm{PbBr}_{3}$ and $\mathrm{MA}_{1-x} \mathrm{FA}_{x} \mathrm{PbBr}_{3}(0 \leq x \leq 1)$. By comparing the solid solutions of the $\mathrm{Az}_{1-x} \mathrm{MA}_{x}$ and $\mathrm{Az}_{1-x} \mathrm{FA}_{x}$ systems, the experimentally-determined range of the $3 \mathrm{C}$ (MA-and FA-rich) solid solution is smaller in $\mathrm{Az}_{1-x} \mathrm{MA}_{x}$, in contrast to the expectation based simply on cation size. In addition, a clear mismatch between the nominal (reaction) and actual (product) composition was found during precipitation synthesis for both $\mathrm{Az}_{1-x} \mathrm{MA}_{x}$ and $\mathrm{Az}_{1-x} \mathrm{FA}_{x}$ systems, indicating that this method is under kinetic control. In comparison, mechanosynthesis is thermodynamically controlled and results in more extensive solid solutions where the nominal composition is retained as the actual composition. Mechanosynthesis has the additional benefit as a (solvent-free) greener approach to study halide perovskite solid solutions.

\section{Results and Discussion}

For mechanosynthesis, samples of $\mathrm{Az}_{1-x} \mathrm{FA}_{x} \mathrm{PbBr}_{3}(0 \leq x \leq 1)$ were prepared by mixing appropriate molar ratios of $\mathrm{AzBr}$ and $\mathrm{FABr}$ with $\mathrm{PbBr}_{2}$ with a mortar and pestle and the mixture was then transferred into the ball mill and ground at $600 \mathrm{rpm}$ for 1 hour. For the antisolvent precipitation synthesis, samples of $\mathrm{Az}_{1-x} \mathrm{FA}_{x} \mathrm{PbBr}_{3}$ were prepared by mixing appropriate molar ratios of $\mathrm{AzBr}$ and $\mathrm{FABr}$ with $\mathrm{PbBr}_{2}$ in a DMF/DMSO (4:1) solution. Acetonitrile was slowly added to the reaction mixture and the resulting precipitate was collected by filtration. Detailed procedures are given in the Supporting Information. During the precipitation synthesis, gradual addition of the 
antisolvent produced a noticeable change in color of the precipitate. Initially, the color of the precipitate was closer to the color of the perovskite with the richer cation source, $\left(\mathrm{AzPbBr}_{3}\right.$ is pale yellow and $\mathrm{FAPbBr}_{3}$ is red-orange) and in later stages of precipitation, the color of the precipitate appeared to be midway between the pale yellow and red-orange colors of the end member compositions. The observation of color progression is difficult during grinding due to the opaque chamber of the ball mill. However, the progress of mechanosynthesis of one sample $\left(\mathrm{Az}_{0.5} \mathrm{FA}_{0.5} \mathrm{PbBr}_{3}\right)$ was tracked by periodic interruption of the grinding process - the color was orange in the early stage of mechanosynthesis and bright yellow in the late stage of grinding (Figure S1). The systematic color change with composition of samples from both synthetic routes is shown in Figures 1a and 1b. Empirically, the transition from yellow to red-orange was observed at a smaller nominal composition $\left(x^{\prime}=0.4\right)$ for the precipitation samples than mechanosynthesized ones $\left(x^{\prime}=0.6\right)$; here, $x^{\prime}$ and $x$ is used to represent the nominal and actual $\mathrm{FA}^{+}$composition in the mixed cation perovskite, respectively.

Comparison of the PXRD data between mechano- and precipitation syntheses is shown in Figures $\mathbf{1 c}$ and 1d. Regions of solid solution formation (single phase regions) separated by a region of twophase mixture at intermediate $x^{\prime}$ can be identified for both syntheses on $\mathrm{Az}_{1-x} \cdot \mathrm{FA}_{x} \cdot \mathrm{PbBr}_{3}$, but the extent of solid solution formation is different. The PXRD of the mechanosynthesized samples indicates that for $x^{\prime} \leq 0.4$ only peaks associated with the $6 \mathrm{H}$ polytype are observed, and for $x^{\prime} \geq$ 0.7 only the $3 \mathrm{C}$ phase is present, while at intermediate $x$ ' values the PXRD indicates a two-phase mixture. The PXRD of the precipitation synthesis samples shows less extensive solid solutions, where for $x^{\prime} \leq 0.2$ or for $x^{\prime} \geq 0.8$ only the $6 \mathrm{H}$ or $3 \mathrm{C}$ polytypes were observed, in comparison to the previously reported $\mathrm{Az}_{1-x} \mathrm{MA}_{x} \mathrm{PbBr}_{3}$ solid solution. ${ }^{21}$ The PXRD peaks for $\mathrm{Az}_{1-x} \cdot \mathrm{FA}_{x} \cdot \mathrm{PbBr}_{3}$ prepared by mechanosynthesis are generally wider than that of the precipitation synthesis, presumably due to the smaller crystallite size. The crystallite size of the $6 \mathrm{H}$ phase in the Az-rich mixture was estimated from the Scherrer equation ${ }^{36}$ (Table S1). The crystallite size of the $6 \mathrm{H}$ phase from the precipitation synthesis is more than twice as large as that from mechanosynthesis, which was confirmed by SEM (Figure S2). However, the crystallite size of 3C phase was hard to determine, especially in the phase mixture region, due to the presence of overlapping major peaks from the $6 \mathrm{H}$ phase. 
a)

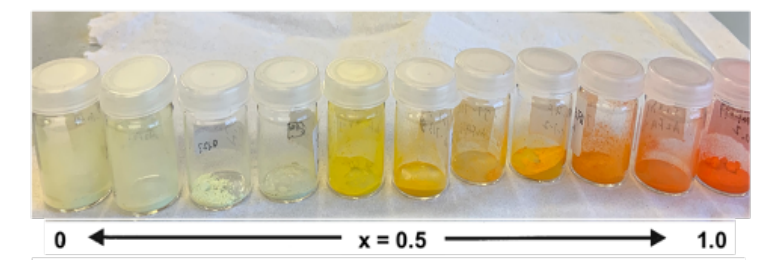

c)

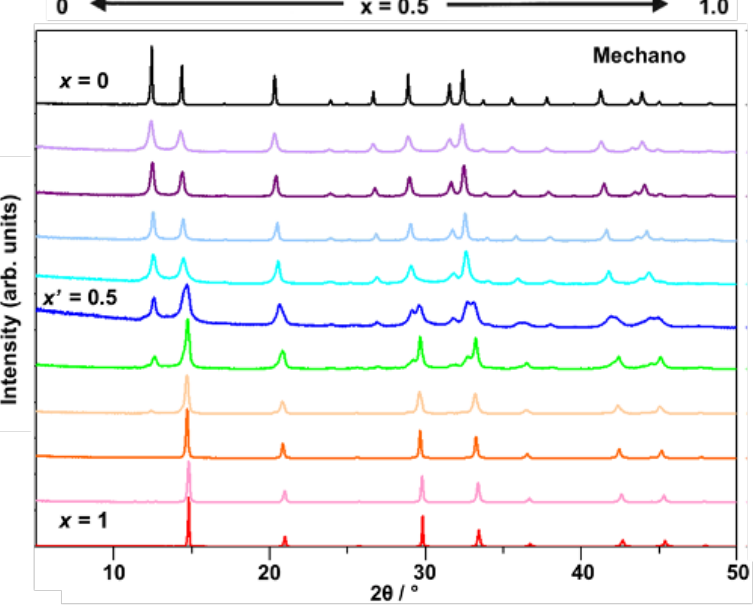

b)

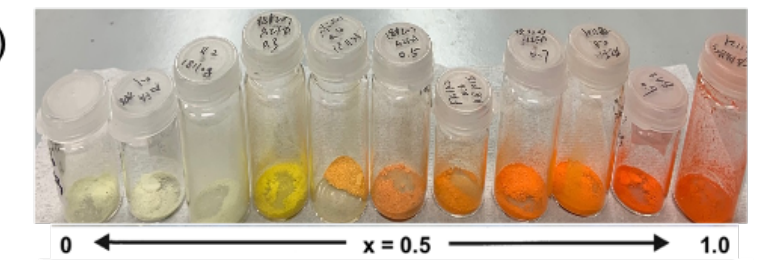

d)

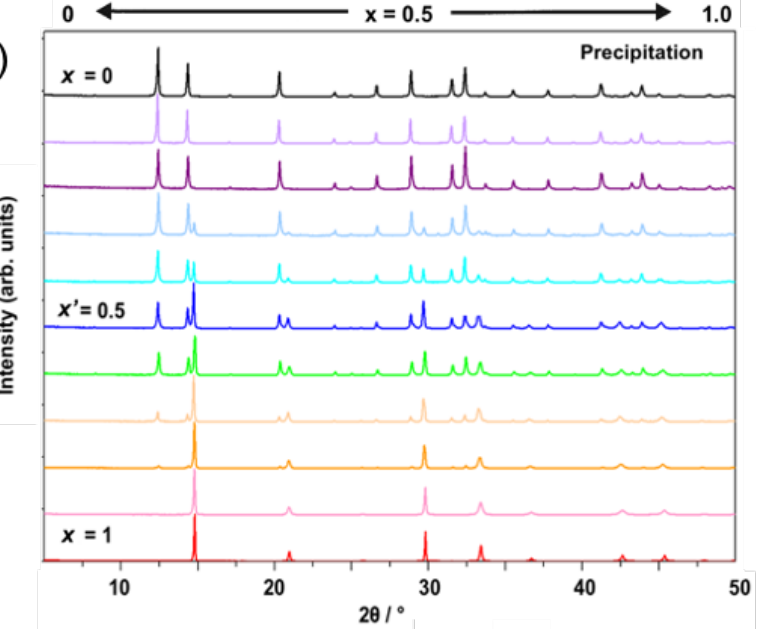

Figure 1. Photos and PXRD data of powders of $\mathrm{Az}_{1-x}, \mathrm{FA}_{x}, \mathrm{PbBr}_{3}$ with nominal composition $0 \leq x$, $\leq 1$ (in $x^{\prime}=0.1$ increments) prepared by (a, c) mechanosynthesis and (b , d) precipitation synthesis.

Using $o$-dichlorobenzene as an internal standard, solution-state ${ }^{1} \mathrm{H}$ NMR analysis (Figure $\mathbf{S 3}$ ) was carried out on the as-synthesized samples to determine the actual $\mathrm{FA}^{+}$composition, $x$, and compare it with nominal $x$, in $\mathrm{Az}_{1-x} \mathrm{FA}_{x} \mathrm{PbBr}_{3}$ prepared by both syntheses routes. The comparison between actual $x$ and nominal $x$ ' is shown in Figure 2. As expected, the actual $x$ obtained by ${ }^{1} \mathrm{H}$ NMR closely matches with nominal $x$ ' in the mechanosynthesis samples as all starting materials are retained during the ball milling reaction. To ensure that this analysis represented the as-synthesised perovskite, ${ }^{1} \mathrm{H}$ NMR analysis of both the isolated bulk powder and the residue left in the ball mill was carried out, with both samples revealing similar compositions (Table S2). By contrast, the ${ }^{1} \mathrm{H}$ NMR analysis of samples from the precipitation synthesis indicated that the actual FA content $(x)$ is consistently lower than the nominal value in the precursor solution $\left(x^{\prime}\right)$. 
a)

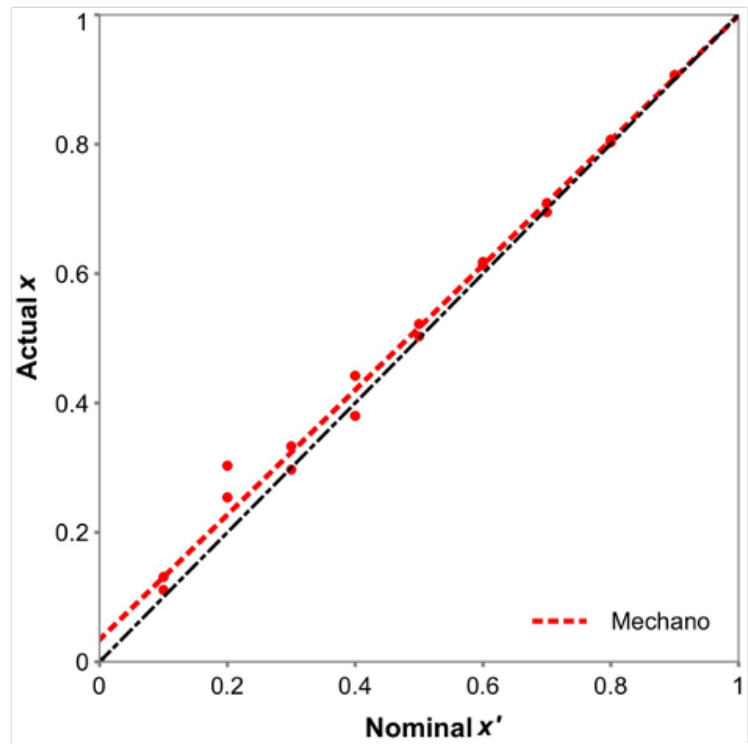

b)

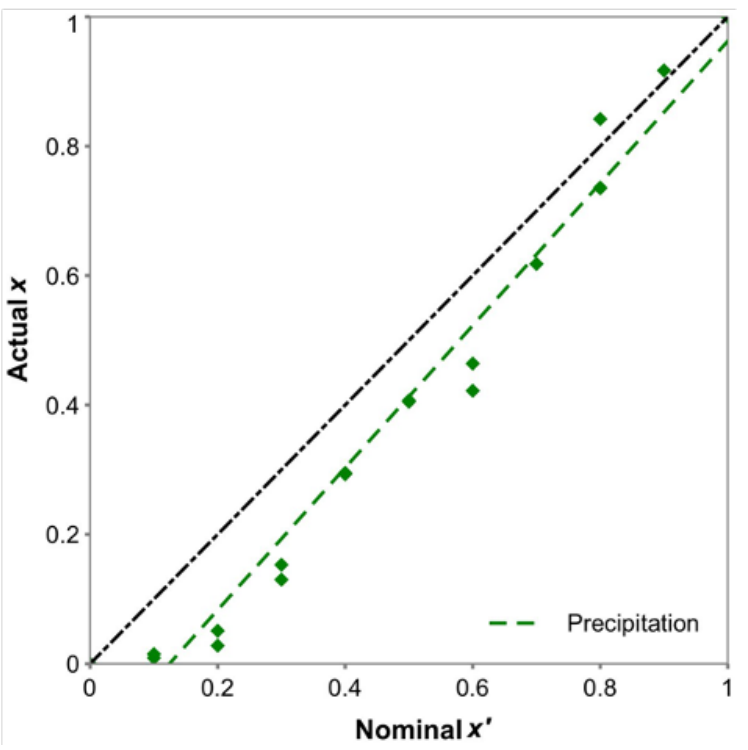

Figure 2. The comparison of nominal ( $\left.x^{\prime}\right)$ and actual $(x) \mathrm{FA}^{+}$cation content determined by ${ }^{1} \mathrm{H}$ NMR analysis on $\mathrm{Az}_{1-x}, \mathrm{FA}_{x}, \mathrm{PbBr}_{3}$ samples prepared by a) mechanosynthesis and b) precipitation synthesis. Dash lines are linear fit to the actual values and black dot-dash lines indicate the instance for $x=x^{\prime}$ composition for comparison.

Rietveld refinement of the PXRD data of samples from both syntheses was carried out to study the phase mixture and the solid solution regions; an example of such a refinement is shown in Figure S4. The weight fraction of both $6 \mathrm{H}$ and $3 \mathrm{C}$ phase was determined from the refinements and are shown in terms of mol fraction versus actual $x$ (obtained by ${ }^{1} \mathrm{H}$ NMR analysis) in Figures 3a and 3b. For samples obtained by mechanosynthesis, an intermediate two-phase region appears for 0.42 $\leq x \leq 0.79$. This contrasts with samples obtained from the precipitation synthesis, where the region is a much larger $0.10 \leq x \leq 0.94$. A rise in temperature of the milling beaker was observed and thus the temperature inside the beaker was measured with a hand-held pyrometer immediately after the grinding. The temperature was found to be $50.4 \pm 3.9{ }^{\circ} \mathrm{C}$. It is possible that the increase in temperature could provide sufficient thermal energy to aid $\mathrm{FA}^{+}$and $\mathrm{Az}^{+}$diffusion into $6 \mathrm{H}$ and $3 \mathrm{C}$ phases during grinding. The observation of the precipitate color change mentioned in the beginning of discussion section, on the other hand, indicates that the precipitation of $6 \mathrm{H}$ and $3 \mathrm{C}$ phases tend to progress at different rates, which is related to their cation concentration in the precursor solution. To investigate the stoichiometry of the A-cation of samples along precipitation progression, a stepwise antisolvent addition experiment was performed (experimental details in Supporting Information). Based on the observed color change during precipitation, the initial assumption was 
that the $6 \mathrm{H}$ phase precipitates first and the $3 \mathrm{C}$ phase follows with increasing volume of antisolvent. The actual $x$ obtained by ${ }^{1} \mathrm{H}$ NMR analysis (Table S2) of the precipitated samples shows that they are deficient in $\mathrm{FA}^{+}$in the early stages of addition of the antisolvent. The incorporation of such a small amount (ca. 6\%) of $\mathrm{FA}^{+}$in the early stages of precipitation is evident from the color of the powders, which appeared close in color to $\mathrm{AzPbBr}_{3}$ (Figure S5). Similarly, there is no 3C phase detected by PXRD in these precipitated samples, the actual $x$ of which is less than 0.07 . This result is consistent with the solid solution/two phase regions indicated in Figure 3. These step-wise antisolvent experiments show that the $6 \mathrm{H}$ phase is more likely to precipitate first with antisolvent present under a similar cation concentration, while the $3 \mathrm{C}$ phase only precipitates with lower $\mathrm{Az}^{+}$ cation concentrations. This kinetic difference accounts for the small solid solution region observed in $\mathrm{Az}_{1-x} \mathrm{FA}_{x} \mathrm{PbBr}_{3}$ samples obtained by precipitation.

In our mixed-cation system $\mathrm{Az}_{1-x} \mathrm{FA}_{x} \mathrm{PbBr}_{3}$, a complete solid solution is impossible as the end members are not isostructural (6H and 3C perovskite), although partial solid solutions may form at either end given the similar A-cation size. In the single phase (solid solution) regions (as determined by PXRD, Figures 3c and 3d), the actual $x$ values as determined from ${ }^{1} \mathrm{H}$ NMR analysis correspond to the actual $\mathrm{FA}^{+}$composition of the solid solution. A more conventional expression of the solid solution general formular is needed as $\mathrm{Az}_{1-y} \mathrm{FA}_{y} \mathrm{PbBr}_{3}\left(6 \mathrm{H}, \mathrm{Az}\right.$-rich) and $\mathrm{Az}_{z} \mathrm{FA}_{1-z} \mathrm{PbBr}_{3}$ (3C, FA-rich), where $y$ and $z$ denote the degree of substitution of FA and Az at the A-site from the parent structure. This also allows us to distinguish them from $\mathrm{Az}_{1-x} \mathrm{FA}_{x} \mathrm{PbBr}_{3}$ especially for mixed phase samples; in the two-phase region, $x$ corresponds to the global FA content with an unknown distribution over the two phases.

The lattice parameters of each polytype as a function of $x$ were determined by Rietveld refinement, including two-phase refinement for intermediate $x$. The cell volume (Figure $\mathbf{3 c}$ and $\mathbf{d}$ ) of $\mathrm{Az}_{1}$ ${ }_{x} \mathrm{FA}_{x} \mathrm{PbBr}_{3}$ as a function of composition shows a close resemblance to the reported $\mathrm{BaTiO}_{3}-$ $\mathrm{CaTiO}_{3}$ solid solution ${ }^{37}$ system, in which the lattice parameter progression shifted from decreasing to invariant as the composition extends from the single phase region into the two-phase region. In the single phase $6 \mathrm{H}$ solid solution region, the lattice constant and cell volume shows a systematic decrease with increasing FA content. This is more evident in samples obtained via mechanosynthesis. For the $3 \mathrm{C}$ region of those samples, the solid solution shows an increase in cell volume with increasing Az content. These volume-composition dependencies suggest that $\mathrm{FA}^{+}$is 
smaller than $\mathrm{Az}^{+}$in contrast to computational studies which suggest they are very similar (actually FA marginally larger). ${ }^{35,38}$ The finding that $\mathrm{FA}^{+}$is smaller than $\mathrm{Az}^{+}$also is consistent with the experimental results that $\mathrm{AzPBBr}_{3}$ forms a $6 \mathrm{H}$ structure and $\mathrm{FAPbBr}_{3}$ forms a cubic structure while their tolerance factors, using computed cation sizes, are both 1.00. The computation of the size effect of organic cations in extended structures is largely based on a hard sphere approximation, which implies their free rotation. This might be not true for either $\mathrm{FA}^{+}$or $\mathrm{Az}^{+}$in these structures at room temperature and requires more study.

For samples obtained from the precipitation synthesis, the cell volume in the Az-rich region is invariant as a function of $x$, which suggests a relatively small degree of substitution by FA when compared to mechosynthesised samples of the same composition. Presumably, this is due to the phase separation between $3 \mathrm{C}$ and $6 \mathrm{H}$ phases during the precipitation as a result of the different precipitation rates. The sharp decrease of the volume at $x=0.84$ is possibly due to the error of the refinement as the fraction of the $6 \mathrm{H}$ phase in these samples is very low. For precipitation synthesized samples in FA-rich region, the slight increase in cell volume indicates a relatively small degree of substitution by Az. Overall, there is limited solid solution formation during the precipitation synthesis. 


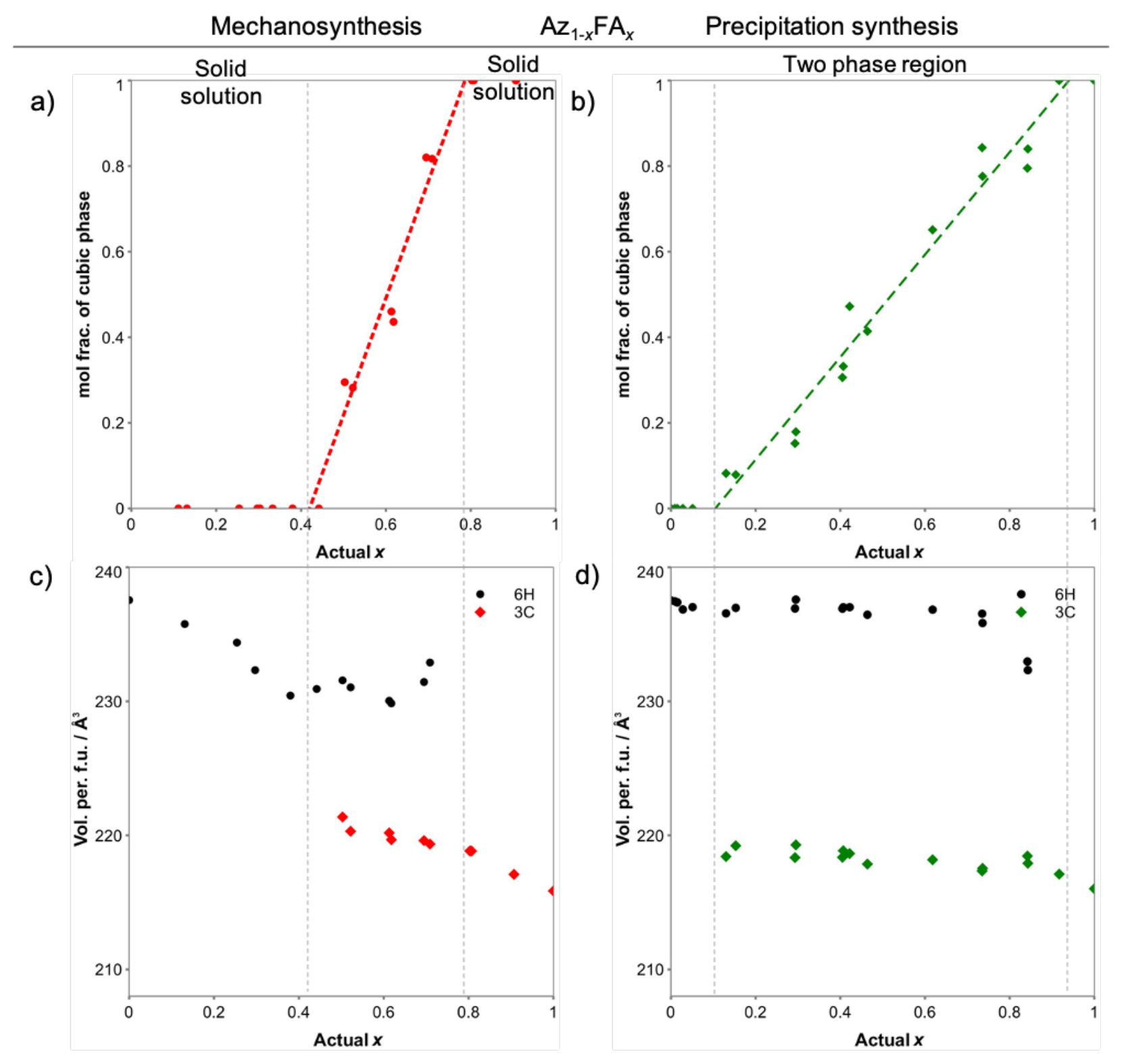

Figure 3. Mole fraction and cell volume (per formula unit) of hexagonal $(6 \mathrm{H})$ and cubic (3C) perovskite phases as determined by Rietveld refinement of $\mathrm{PXRD}$ data for $\mathrm{Az}_{1-x} \mathrm{FA}_{x} \mathrm{PbBr}_{3} \mathrm{samples}$ prepared by mechano- $(a, c)$ and precipitation synthesis $(b, d)$. Values are shown as a function of overall, actual $x$ as determined by NMR. The vertical dashed lines indicate the boundaries between the single phase solid solution and intermediate two-phase regions.

To further study the competitive solid solution formation, we applied similar synthesis protocols to the $\mathrm{Az}_{1-x} \mathrm{MA}_{x} \mathrm{PbBr}_{3}$ and $\mathrm{MA}_{1-x} \mathrm{FA}_{x} \mathrm{PbBr}_{3}$ systems $(0 \leq x \leq 1)$. The compositional analysis of $\mathrm{Az}_{1-}$ 
${ }_{x} \mathrm{MA}_{x} \mathrm{PbBr}_{3}$ reveals a similar result to the $\mathrm{Az}_{1-x} \mathrm{FA}_{x}$ system (Figure S6), where (actual) $x \approx$ (nominal) $x$ ' for the mechanosynthesis and $x<x$ ' for the precipitation synthesis. $\mathrm{MA}_{1-x} \mathrm{FA}_{x} \mathrm{PbBr}_{3}$, by contrast, shows $x \approx x$ ' for both synthetic routes and Rietveld refinement of PXRD data shows that the cell volume varies linearly as a function of actual $x$ (Figure S7). The data for the precipitation synthesis of $\mathrm{Az}_{1-x} \mathrm{MA}_{x} \cdot \mathrm{PbBr}_{3}$ was taken from our previous study, ${ }^{21}$ but this time replotted based on actual $x$. Rietveld refinements for the $\mathrm{Az}_{1-x} \mathrm{MA}_{x}$ system shows the presence of limited solid solutions of composition $\mathrm{Az}_{1-y} \mathrm{MA}_{y} \mathrm{PbBr}_{3}(6 \mathrm{H})$ and $\mathrm{Az}_{z} \mathrm{MA}_{1-z} \mathrm{PbBr}_{3}$ (3C) for both syntheses (Figure 4). For samples obtained from the mechanosynthesis, an intermediate two-phase region appears for $0.27 \leq x \leq 0.49$, compared to precipitation synthesized samples, where the region is larger $(0.07 \leq x \leq 0.71)$. The cell volume expansion as a function of increasing Az content (fitted lines) in the $3 \mathrm{C}$ solid solution region is similar for both mechano- and precipitation syntheses. 

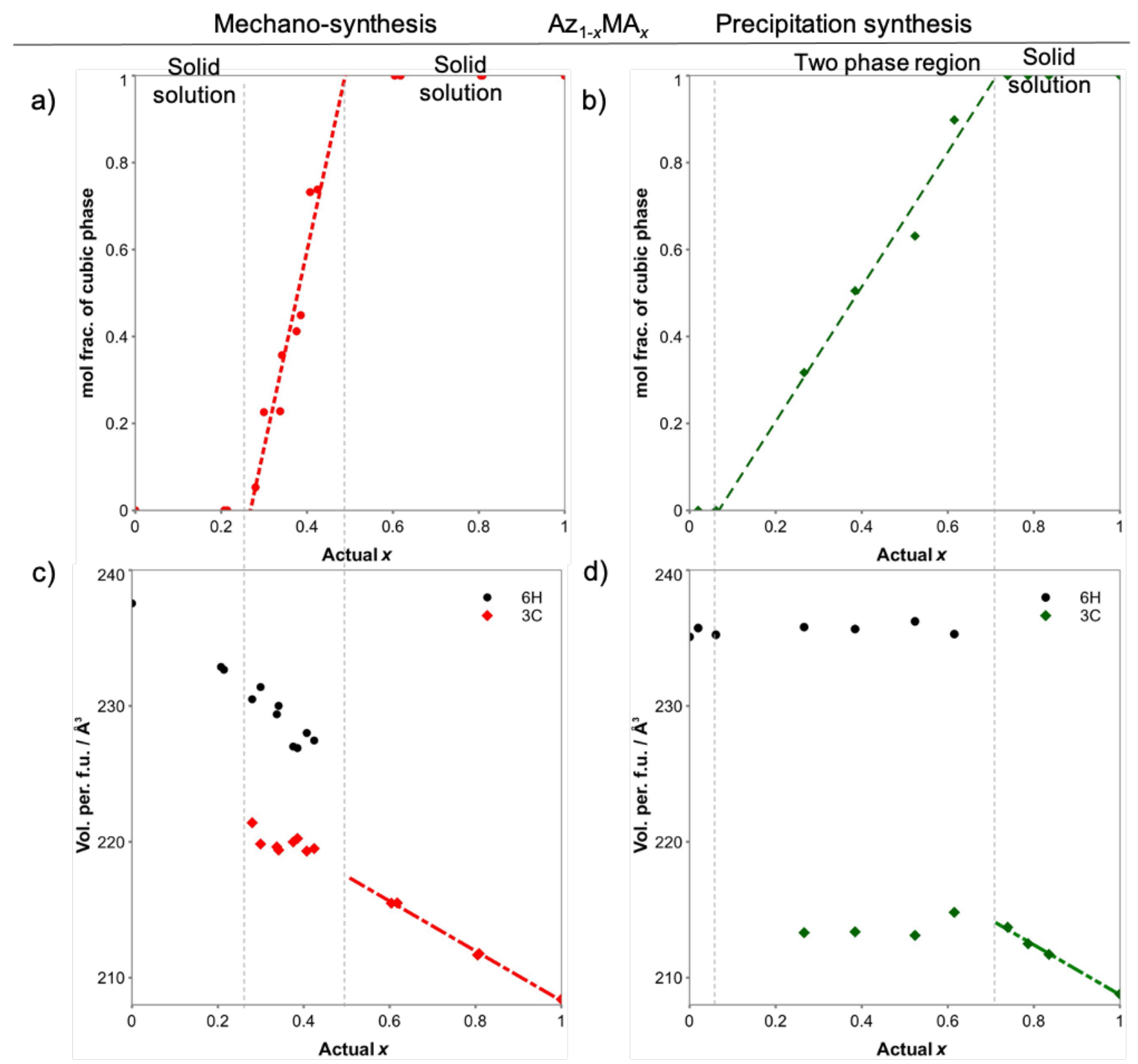

d)

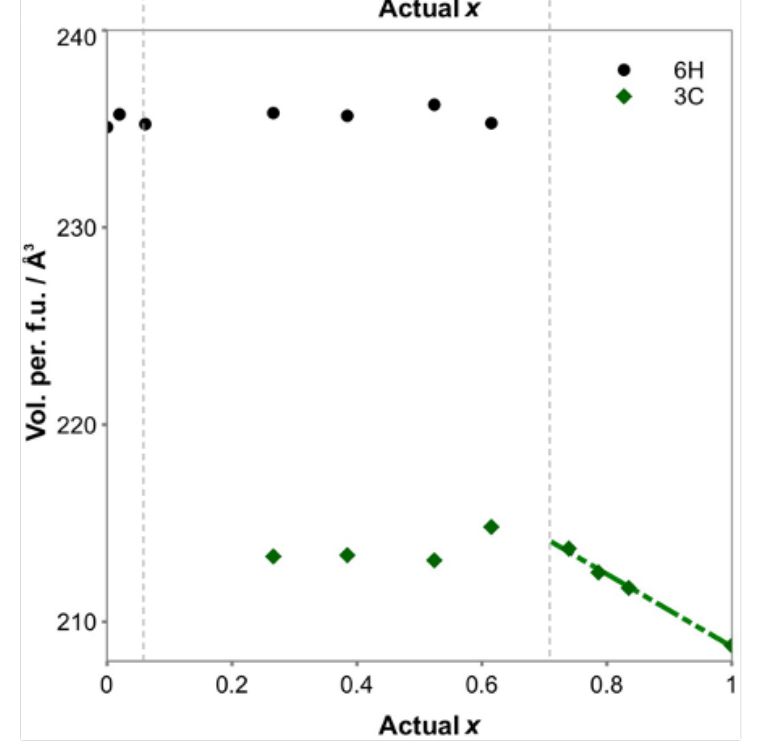

Figure 4. Mole fraction and cell volume (per formula unit) of hexagonal $(6 \mathrm{H})$ and cubic (3C) perovskite phases as determined by Rietveld refinement of PXRD data for $\mathrm{Az}_{1-x} \mathrm{MA}_{x} \mathrm{PbBr}_{3} \mathrm{samples}$ prepared by mechano- (a, c) and precipitation synthesis $(b, d)$. Values are shown as a function of (overall) actual $x$ as determined by NMR. The vertical dashed lines indicate the boundaries between the single phase solid solution and intermediate two-phase regions. The dash-dot lines and dash-dot-dot lines are linear fits to the cell volume as a function of actual $x$ for mechano- and precipitation samples, respectively in the $3 \mathrm{C}$ solid solution region. 
The solid solution ranges of the $\mathrm{Az}_{1-x} \mathrm{MA}_{x}$ and $\mathrm{Az}_{1-x} \mathrm{FA}_{x}$ systems are included in Table 1. Our experimental results indicate that the size of $\mathrm{Az}^{+}$is larger than that of $\mathrm{FA}^{+}$, and both cations are larger than $\mathrm{MA}^{+}$. The relative size relationship between $\mathrm{FA}^{+}$and $\mathrm{MA}^{+}$can be confirmed from the lattice parameters of the reported (cubic) $\mathrm{FAPbBr}_{3}$ and $\mathrm{MAPbBr}_{3}$, where $a\left(\mathrm{FAPbBr}_{3}\right)=5.98 \AA$ and $a\left(\mathrm{MAPbBr}_{3}\right)=5.92 \AA . .^{21,39}$ Logically, it would be easier for relatively larger $\mathrm{Az}^{+}$to replace the cations in the larger A-site interspace of $\mathrm{FAPbBr}_{3}$ independent of the synthetic method. However, the observations here are contrary to this notion: for mechanosynthesis of the $3 \mathrm{C}$ solid solutions, in $\mathrm{Az}_{z} \mathrm{FA}_{1-z} \mathrm{PbBr}_{3}$, the degree of $\mathrm{Az}$ substitution extends to $z=0.21$, compared to $z=0.51$ for $\mathrm{Az}_{z} \mathrm{MA}_{1-z} \mathrm{PbBr}_{3}$. (Precipitation synthesized samples show similar results where the solid solution range of $\mathrm{Az}_{z} \mathrm{FA}_{1-z} \mathrm{PbBr}_{3}$ is smaller than that of $\mathrm{Az}_{z} \mathrm{MA}_{1-z} \mathrm{PbBr}_{3}$, but this observation does not take into account any possible kinetically limited aspects). Of the two synthetic methods the mechanosynthesis is expected to produce thermodynamically stable products. It is unexpected that the experimentally-determined solid solution range is in contrast to that predicted by cation size.

The $6 \mathrm{H}$ solid solution ranges described by $\mathrm{Az}_{1-y} \mathrm{FA}_{y} \mathrm{PbBr}_{3}$ and $\mathrm{Az}_{1-y} \mathrm{MA}_{y} \mathrm{PbBr}_{3}$ are both extremely limited in the precipitation synthesized samples. For the mechanosynthesized $6 \mathrm{H}$ solid solution, $\mathrm{Az}_{1-y} \mathrm{FA}_{y} \mathrm{PbBr}_{3}$, the degree of $\mathrm{FA}$ substitution extends to $y \leq 0.42$ and to $y \leq 0.27$ for $\mathrm{Az}_{1}$ ${ }_{y} \mathrm{MA}_{y} \mathrm{PbBr}_{3}$, which are consistent with size predictions. In other words, the replacement of $\mathrm{Az}^{+}$in the $6 \mathrm{H}$-structured solid solutions favors the cation of similar size $\left(\mathrm{FA}^{+}\right)$than the smaller cation $\left(\mathrm{MA}^{+}\right)$.

Table 1. Solid solution ranges for $A z_{1-x} \mathrm{MA}_{x}$ and $\mathrm{Az}_{1-x} \mathrm{FA}_{x}$ samples prepared by mechano- and precipitation syntheses.

\begin{tabular}{cccc}
\hline \multirow{2}{*}{$6 \mathrm{c}$} & $\begin{array}{c}\text { Solid solution } \\
\text { range }\end{array}$ & $\begin{array}{c}\text { Mechano- } \\
\text { synthesis }\end{array}$ & $\begin{array}{c}\text { Precipitation } \\
\text { synthesis }\end{array}$ \\
\hline \multirow{2}{*}{$3 \mathrm{H}$} & $\mathrm{Az}_{1-y} \mathrm{FA}_{y} \mathrm{PbBr}_{3}$ & $y \leq 0.42$ & $y \leq 0.10$ \\
\cline { 2 - 4 } & $\mathrm{Az}_{1-y} \mathrm{MA}_{y} \mathrm{PbBr}_{3}$ & $y \leq 0.27$ & $y \leq 0.07$ \\
\hline \multirow{2}{*}{$\mathrm{Az}_{z} \mathrm{FA}_{1-z} \mathrm{PbBr}_{3}$} & $z \leq 0.21$ & $z \leq 0.06$ \\
\cline { 2 - 4 } & $\mathrm{Az}_{z} \mathrm{MA}_{1-z} \mathrm{PbBr}_{3}$ & $z \leq 0.51$ & $z \leq 0.29$ \\
\hline
\end{tabular}


The optical properties of the solid solutions/two-phase mixtures of $\mathrm{Az}_{1-x} \mathrm{FA}_{x} \mathrm{PbBr}_{3}$ samples were studied by absorption spectroscopy (Figure 5). The absorption onsets are systematically red-shifted with increasing $\mathrm{FA}^{+}$, regardless of how the samples were synthesized. Optical measurements indicate that the absorption edge for the $6 \mathrm{H}$ solid solution samples obtained from the mechanosynthesis is red-shifted from $c a .440 \mathrm{~nm}\left(\mathrm{AzPBBr}_{3}\right)$ to $c a .540 \mathrm{~nm}(y=0.38)$. The absorption edge of $\mathrm{Az}_{0.62} \mathrm{FA}_{0.38} \mathrm{PbBr}_{3}$ is relatively close to that of $\mathrm{FAPbBr}_{3}(585 \mathrm{~nm})$. As the solidstate absorption spectra is surface sensitive, the shifted absorption edge at such composition may be due to the presence of some $3 \mathrm{C}$ crystallites formed at the surface, the amount of which is insufficient to be detected by PXRD. Moreover, the absorption edge for the $6 \mathrm{H}$ solid solution samples obtained from the precipitation synthesis remains invariant at $c a .440 \mathrm{~nm}(0 \leq y \leq 0.051)$. By contrast, the $3 \mathrm{C}$ solid solution from both syntheses shows a blue-shift from $c a .585 \mathrm{~nm}$ $\left(\mathrm{FAPbBr}_{3}\right)$ to $c a .565 \mathrm{~nm}(z=0.20$, mechanosynthesis $)$ and $c a .576 \mathrm{~nm}(z=0.08$, precipitation synthesis), respectively. In the two-phase region $(0.10 \leq x \leq 0.94)$ of the precipitation synthesised samples, the low volume fraction of the $6 \mathrm{H}$ phase for $0.62 \leq x \leq 0.74$ (Figure $2 \mathbf{b}$ ) means that the absorption is dominated by the $3 \mathrm{C}$ component and the absorption edge remains around $560 \mathrm{~nm}$. For the $0.14 \leq x<0.62$ compositions, which have a significant phase fraction of both $6 \mathrm{H}$ and $3 \mathrm{C}$, two absorption edges were observed corresponding to each phase $(6 \mathrm{H} \sim 470 \mathrm{~nm} ; 3 \mathrm{C} \sim 560 \mathrm{~nm})$. The absorption spectra of the corresponding $A z_{1-x} \mathrm{MA}_{x}$ samples are shown in Figure $\mathbf{S 8}$ as a comparison, and show a similar absorption edge shift as a function of $x$.

The band gap values calculated from the onset of the absorption spectra from both $\mathrm{Az}_{1-x} \mathrm{FA}_{x}$ and $A z_{1-x} M_{A}$ samples are shown in Figure S9. The bandgap of the $\mathrm{Az}_{1-x} \mathrm{FA}_{x}$ samples in the $6 \mathrm{H}$ solid solution region shows a nonlinear character, which can be rationalized in terms of the bowing effect, ${ }^{37,40}$ where a lower bandgap was attained for the intermediate composition of solid solutions than expected from linear interpolation of the end member values. The bandgap of the $3 \mathrm{C}$ solid solution and two-phase region samples show a progressive increase with increasing Az content (decreasing $x$ ). This trend is due to the unit cell expansion as a result of elongation of the $\mathrm{Pb}-\mathrm{Br}$ bond, as discussed in our previous study. ${ }^{21}$ 
a)

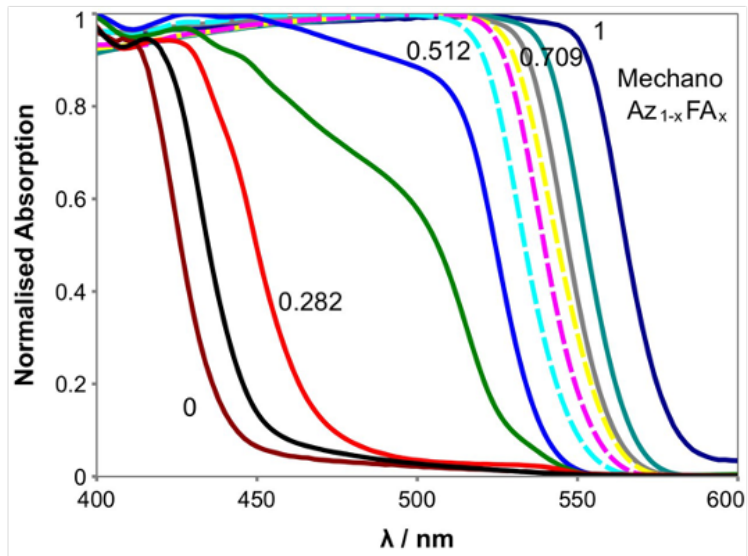

b)

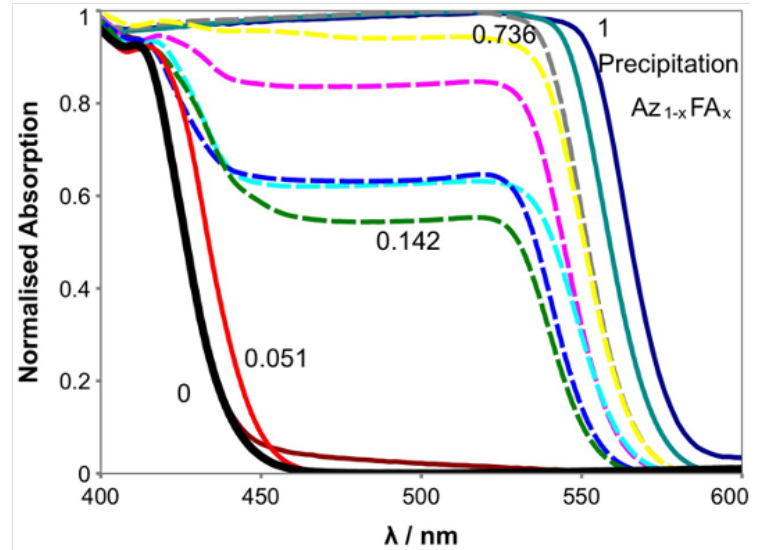

Figure 5. Absorption spectra of $\mathrm{Az}_{1-x} \mathrm{FA}_{x} \mathrm{PbBr}_{3}$ samples prepared by a) mechanosynthesis b) precipitation synthesis. Spectra from single phase (solid solution) compositions as determined by PXRD are plotted with solid lines and multi-phase samples with dashed lines.

\section{Conclusions}

Solid solution formation in the system $\mathrm{Az}_{1-x} \mathrm{FA}_{x} \mathrm{PbBr}_{3}(0 \leq x \leq 1)$ was explored using both mechano- and precipitation syntheses. For samples obtained from the precipitation synthesis, the actual $\mathrm{FA} \%$ in the precipitate was found to be less than the nominal composition in the reaction solution as a result of different precipitation rates of $3 \mathrm{C}$ and $6 \mathrm{H}$ perovskite polytypes. No such composition mismatch was found for mechanosynthesized samples. A 3C-6H two phase region was found to be present for $0.42 \leq x \leq 0.79$ and $0.10 \leq x \leq 0.94$, for mechano- and precipitation synthesis of $\mathrm{Az}_{1-x} \mathrm{FA}_{x} \mathrm{PbBr}_{3}$, respectively. The cell volume dependence on the composition (volume decreases with increasing FA content) in both 6H (Az-rich) and 3C (FA-rich) solid solution regions suggests the $\mathrm{FA}^{+}$cation is actually smaller than $\mathrm{Az}^{+}$, which is incongruent to the cation size reported in the literature. ${ }^{35,38}$ By comparison, in the $3 \mathrm{C}$ solid solution regions (Az-poor), the solid solution range in $\mathrm{Az}_{z} \mathrm{FA}_{1-z} \mathrm{PbBr}_{3}$ is surprisingly smaller than in $\mathrm{Az}_{z} \mathrm{MA}_{1-z} \mathrm{PbBr}_{3}$, given that $\mathrm{MA}^{+}$is much smaller than $\mathrm{FA}^{+}$. It seems other factors also contribute to the solid solution formation of organic-inorganic hybrid perovskites, not simply the size of A-site cation. Overall, our study reveals the importance of cross-checking the nominal (reaction) with the actual (product) composition, especially when the synthetic method may be under kinetic control or multi phases can be formed in the system. The computed size of organic cations and the presence of any 
dynamic/preferential bonding effects should be re-examined and considered during study of organic-inorganic hybrid perovskite systems.

Associated content

\section{Supporting Information}

The Supporting Information contains Experimental details, SEM, details of ${ }^{1} \mathrm{H}$ NMR analysis, examples of Rietveld refinement and absorption spectra. The research data supporting this publication can be accessed at [].

\section{Author information}

Corresponding authors:

eli.zysman-colman@st-andrews.ac.uk

finlay.morrison@st-andrews.ac.uk

\section{Acknowledgements}

We thank the Chinese Scholarship Council and the University of St Andrews for Ph.D. Studentship support (to JT, CSC No. 201603780020).

\section{References}

(1) Hou, Y.; Aydin, E.; De Bastiani, M.; Xiao, C.; Isikgor, F. H.; Xue, D.-J.; Chen, B.; Chen, H.; Bahrami, B.; Chowdhury, A. H.; et al. Efficient Tandem Solar Cells with SolutionProcessed Perovskite on Textured Crystalline Silicon. Science (80-. ). 2020, 367 (6482), 1135-1140.

(2) Alsalloum, A. Y.; Turedi, B.; Zheng, X.; Mitra, S.; Zhumekenov, A. A.; Lee, K. J.; Maity, P.; Gereige, I.; AlSaggaf, A.; Roqan, I. S.; et al. Low-Temperature Crystallization Enables 21.9\% Efficient Single-Crystal MAPbI 3 Inverted Perovskite Solar Cells. ACS Energy Lett. 2020, 5 (2), 657-662.

(3) Liu, J.; Han, Q.; Bai, Y.; Du, K. Z.; Li, T.; Ji, D.; Zhou, Y.; Cao, C.; Shin, D.; Ding, J.; et 
al. Additive Engineering for High-Performance Room-Temperature-Processed Perovskite Absorbers with Micron-Size Grains and Microsecond-Range Carrier Lifetimes. Energy Environ. Sci. 2017, 10 (11), 2365-2371.

(4) Liang, H.; Yuan, F.; Johnston, A.; Gao, C.; Choubisa, H.; Gao, Y.; Wang, Y.; Sagar, L. K.; Sun, B.; Li, P.; et al. High Color Purity Lead-Free Perovskite Light-Emitting Diodes via Sn Stabilization. Adv. Sci. 2020, 7 (8), 1903213.

(5) Ma, D.; Todorović, P.; Meshkat, S.; Saidaminov, M. I.; Wang, Y.-K.; Chen, B.; Li, P.; Scheffel, B.; Quintero-Bermudez, R.; Fan, J. Z.; et al. Chloride Insertion-Immobilization Enables Bright, Narrowband, and Stable Blue-Emitting Perovskite Diodes. J. Am. Chem. Soc. 2020, 142 (11), 5126-5134.

(6) Jia, Y.; Kerner, R. A.; Grede, A. J.; Rand, B. P.; Giebink, N. C. Continuous-Wave Lasing in an Organic-Inorganic Lead Halide Perovskite Semiconductor. Nat. Photonics 2017, 11 (12), 784-788.

(7) Cao, M.; Tian, J.; Cai, Z.; Peng, L.; Yang, L.; Wei, D. Perovskite Heterojunction Based on CH3NH3PbBr3 Single Crystal for High-Sensitive Self-Powered Photodetector. Appl. Phys. Lett. 2016, 109 (23), 233303.

(8) Zhou, H.; Song, Z.; Grice, C. R.; Chen, C.; Yang, X.; Wang, H.; Yan, Y. Pressure-Assisted Annealing Strategy for High-Performance Self-Powered All-Inorganic Perovskite Microcrystal Photodetectors. J. Phys. Chem. Lett. 2018, 9 (16), 4714-4719.

(9) Stoumpos, C. C.; Malliakas, C. D.; Kanatzidis, M. G. Semiconducting Tin and Lead Iodide Perovskites with Organic Cations: Phase Transitions, High Mobilities, and near-Infrared Photoluminescent Properties. Inorg. Chem. 2013, 52 (15), 9019-9038.

(10) Kojima, A.; Teshima, K.; Shirai, Y.; Miyasaka, T. Organometal Halide Perovskites as Visible-Light Sensitizers for Photovoltaic Cells. J. Am. Chem. Soc. 2009, 131 (17), 60506051.

(11) Levchuk, I.; Osvet, A.; Tang, X.; Brandl, M.; Perea, J. D.; Hoegl, F.; Matt, G. J.; Hock, R.; Batentschuk, M.; Brabec, C. J. Brightly Luminescent and Color-Tunable Formamidinium Lead Halide Perovskite FAPbX 3 (X = Cl, Br, I) Colloidal Nanocrystals. Nano Lett. 2017, 
$17(5), 2765-2770$.

(12) Noh, J. H.; Im, S. H.; Heo, J. H.; Mandal, T. N.; Seok, S. Il. Chemical Management for Colorful, Efficient, and Stable Inorganic-Organic Hybrid Nanostructured Solar Cells. Nano Lett. 2013, 13 (4), 1764-1769.

(13) Chen, D.; Li, J.; Chen, X.; Chen, J.; Zhong, J. Grinding Synthesis of APbX 3 (A = MA, FA, Cs; $\mathrm{X}=\mathrm{Cl}, \mathrm{Br}$, I) Perovskite Nanocrystals. ACS Appl. Mater. Interfaces 2019, 11 (10), 10059-10067.

(14) Zhu, H.; Fu, Y.; Meng, F.; Wu, X.; Gong, Z.; Ding, Q.; Gustafsson, M. V.; Trinh, M. T.; Jin, S.; Zhu, X. Y. Lead Halide Perovskite Nanowire Lasers with Low Lasing Thresholds and High Quality Factors. Nat. Mater. 2015, 14 (6), 636-642.

(15) Spanopoulos, I.; Hadar, I.; Ke, W.; Tu, Q.; Chen, M.; Tsai, H.; He, Y.; Shekhawat, G.; Dravid, V. P.; Wasielewski, M. R.; et al. Uniaxial Expansion of the 2D Ruddlesden-Popper Perovskite Family for Improved Environmental Stability. J. Am. Chem. Soc. 2019, 141 (13), $5518-5534$.

(16) Zhao, Y.; Xu, X.; You, X. Colloidal Organometal Halide Perovskite (MAPbBrxI3-x, $0 \leq \mathrm{x} \leq 3)$ Quantum Dots: Controllable Synthesis and Tunable Photoluminescence. Sci. Rep. 2016, $6(1), 35931$.

(17) Freppon, D. J.; Men, L.; Burkhow, S. J.; Petrich, J. W.; Vela, J.; Smith, E. A. Photophysical Properties of Wavelength-Tunable Methylammonium Lead Halide Perovskite Nanocrystals. J. Mater. Chem. C 2017, 5 (1), 118-126.

(18) Soe, C. M. M.; Stoumpos, C. C.; Kepenekian, M.; Traoré, B.; Tsai, H.; Nie, W.; Wang, B.; Katan, C.; Seshadri, R.; Mohite, A. D.; et al. New Type of 2D Perovskites with Alternating Cations in the Interlayer Space, $\left(\mathrm{C}\left(\mathrm{NH}_{2}\right)_{3}\right)\left(\mathrm{CH}_{3} \mathrm{NH}_{3}\right){ }_{n} \mathrm{~Pb}_{n} \mathrm{I}_{3}{ }_{n+1}$ : Structure, Properties, and Photovoltaic Performance. J. Am. Chem. Soc. 2017, 139 (45), 16297-16309.

(19) Stoumpos, C. C.; Cao, D. H.; Clark, D. J.; Young, J.; Rondinelli, J. M.; Jang, J. I.; Hupp, J. T.; Kanatzidis, M. G. Ruddlesden-Popper Hybrid Lead Iodide Perovskite 2D Homologous Semiconductors. Chem. Mater. 2016, 28 (8), 2852-2867.

(20) Cao, D. H.; Stoumpos, C. C.; Farha, O. K.; Hupp, J. T.; Kanatzidis, M. G. 2D Homologous 
Perovskites as Light-Absorbing Materials for Solar Cell Applications. J. Am. Chem. Soc. 2015, 137 (24), 7843-7850.

(21) Tian, J.; Cordes, D. B.; Quarti, C.; Beljonne, D.; Slawin, A. M. Z.; Zysman-Colman, E.; Morrison, F. D. Stable 6H Organic-Inorganic Hybrid Lead Perovskite and Competitive Formation of $6 \mathrm{H}$ and 3C Perovskite Structure with Mixed A Cations. ACS Appl. Energy Mater. 2019, 2 (8), 5427-5437.

(22) Prochowicz, D.; Yadav, P.; Saliba, M.; Saski, M.; Zakeeruddin, S. M.; Lewiński, J.; Grätzel, M. Mechanosynthesis of Pure Phase Mixed-Cation MA: XFA1- XPbI3 Hybrid Perovskites: Photovoltaic Performance and Electrochemical Properties. Sustain. Energy Fuels 2017, 1 (4), 689-693.

(23) Azam, M.; Yue, S.; Xu, R.; Liu, K.; Ren, K.; Sun, Y.; Liu, J.; Wang, Z.; Qu, S.; Lei, Y.; et al. Highly Efficient Solar Cells Based on Cl Incorporated Tri-Cation Perovskite Materials. J. Mater. Chem. A 2018, 6 (28), 13725-13734.

(24) Ng, M.; Halpert, J. E. Single Crystals of Mixed Br/Cl and Sn-Doped Formamidinium Lead Halide Perovskites via Inverse Temperature Crystallization. RSC Adv. 2020, 10 (7), 38323836.

(25) Zhang, X.; Li, L.; Sun, Z.; Luo, J. Rational Chemical Doping of Metal Halide Perovskites. Chem. Soc. Rev. 2019, 48 (2), 517-539.

(26) Saliba, M.; Matsui, T.; Domanski, K.; Seo, J.-Y.; Ummadisingu, A.; Zakeeruddin, S. M.; Correa-Baena, J.-P.; Tress, W. R.; Abate, A.; Hagfeldt, A.; et al. Incorporation of Rubidium Cations into Perovskite Solar Cells Improves Photovoltaic Performance. Science (80-. ). 2016, 354 (6309), 206-209.

(27) Shi, D.; Adinolfi, V.; Comin, R.; Yuan, M.; Alarousu, E.; Buin, A.; Chen, Y.; Hoogland, S.; Rothenberger, A.; Katsiev, K.; et al. Solar Cells. Low Trap-State Density and Long Carrier Diffusion in Organolead Trihalide Perovskite Single Crystals. Science 2015, 347 (6221), 519-522.

(28) Martínez-Sarti, L.; Palazon, F.; Sessolo, M.; Bolink, H. J. Dry Mechanochemical Synthesis of Highly Luminescent, Blue and Green Hybrid Perovskite Solids. Adv. Opt. Mater. 2020, 
$8(4), 1901494$.

(29) Abdelhady, A. L.; Saidaminov, M. I.; Murali, B.; Adinolfi, V.; Voznyy, O.; Katsiev, K.; Alarousu, E.; Comin, R.; Dursun, I.; Sinatra, L.; et al. Heterovalent Dopant Incorporation for Bandgap and Type Engineering of Perovskite Crystals. J. Phys. Chem. Lett. 2016, 7 (2), $295-301$.

(30) Zhang, Z.; Ren, L.; Yan, H.; Guo, S.; Wang, S.; Wang, M.; Jin, K. Bandgap Narrowing in Bi-Doped CH 3 NH 3 PbCl 3 Perovskite Single Crystals and Thin Films. J. Phys. Chem. C 2017, 121 (32), 17436-17441.

(31) Nayak, P. K.; Sendner, M.; Wenger, B.; Wang, Z.; Sharma, K.; Ramadan, A. J.; Lovrinčić, R.; Pucci, A.; Madhu, P. K.; Snaith, H. J. Impact of Bi 3+ Heterovalent Doping in OrganicInorganic Metal Halide Perovskite Crystals. J. Am. Chem. Soc. 2018, 140 (2), 574-577.

(32) van der Stam, W.; Geuchies, J. J.; Altantzis, T.; van den Bos, K. H. W.; Meeldijk, J. D.; Van Aert, S.; Bals, S.; Vanmaekelbergh, D.; de Mello Donega, C. Highly Emissive Divalent-Ion-Doped Colloidal CsPb 1-x M x Br 3 Perovskite Nanocrystals through Cation Exchange. J. Am. Chem. Soc. 2017, 139 (11), 4087-4097.

(33) Weber, O. J.; Charles, B.; Weller, M. T. Phase Behaviour and Composition in the Formamidinium-Methylammonium Hybrid Lead Iodide Perovskite Solid Solution. J. Mater. Chem. A 2016, 4 (40), 15375-15382.

(34) Ito, N.; Kamarudin, M. A.; Hirotani, D.; Zhang, Y.; Shen, Q.; Ogomi, Y.; Iikubo, S.; Minemoto, T.; Yoshino, K.; Hayase, S. Mixed Sn-Ge Perovskite for Enhanced Perovskite Solar Cell Performance in Air. J. Phys. Chem. Lett. 2018, 9 (7), 1682-1688.

(35) Kieslich, G.; Sun, S.; Cheetham, A. K.; Cheetham, T.; Gregor, K.; Shijing, S.; Anthony, K. C. Solid-State Principles Applied to Organic-Inorganic Perovskites: New Tricks for an Old Dog. Chem. Sci. 2014, 5 (12), 4712-4715.

(36) Langford, J. I.; Wilson, A. J. C. Scherrer after Sixty Years: A Survey and Some New Results in the Determination of Crystallite Size. J. Appl. Crystallogr. 1978, 11 (2), 102-113.

(37) Lee, S.; Levi, R. D.; Qu, W.; Lee, S. C.; Randall, C. A. Band-Gap Nonlinearity in Perovskite Structured Solid Solutions. J. Appl. Phys. 2010, 107 (2), 1-6. 
(38) Becker, M.; Klüner, T.; Wark, M. Formation of Hybrid ABX 3 Perovskite Compounds for Solar Cell Application: First-Principles Calculations of Effective Ionic Radii and Determination of Tolerance Factors. Dalt. Trans. 2017, 46 (11), 3500-3509.

(39) Schueller, E. C.; Laurita, G.; Fabini, D. H.; Stoumpos, C. C.; Kanatzidis, M. G.; Seshadri, R. Crystal Structure Evolution and Notable Thermal Expansion in Hybrid Perovskites Formamidinium Tin Iodide and Formamidinium Lead Bromide. Inorg. Chem. 2018, 57 (2), 695-701.

(40) Chatterjee, S.; Payne, J.; Irvine, J. T. S.; Pal, A. J. Bandgap Bowing in a Zero-Dimensional Hybrid Halide Perovskite Derivative: Spin-Orbit Coupling: Versus Lattice Strain. J. Mater. Chem. A 2020, 8 (8), 4416-4427. 

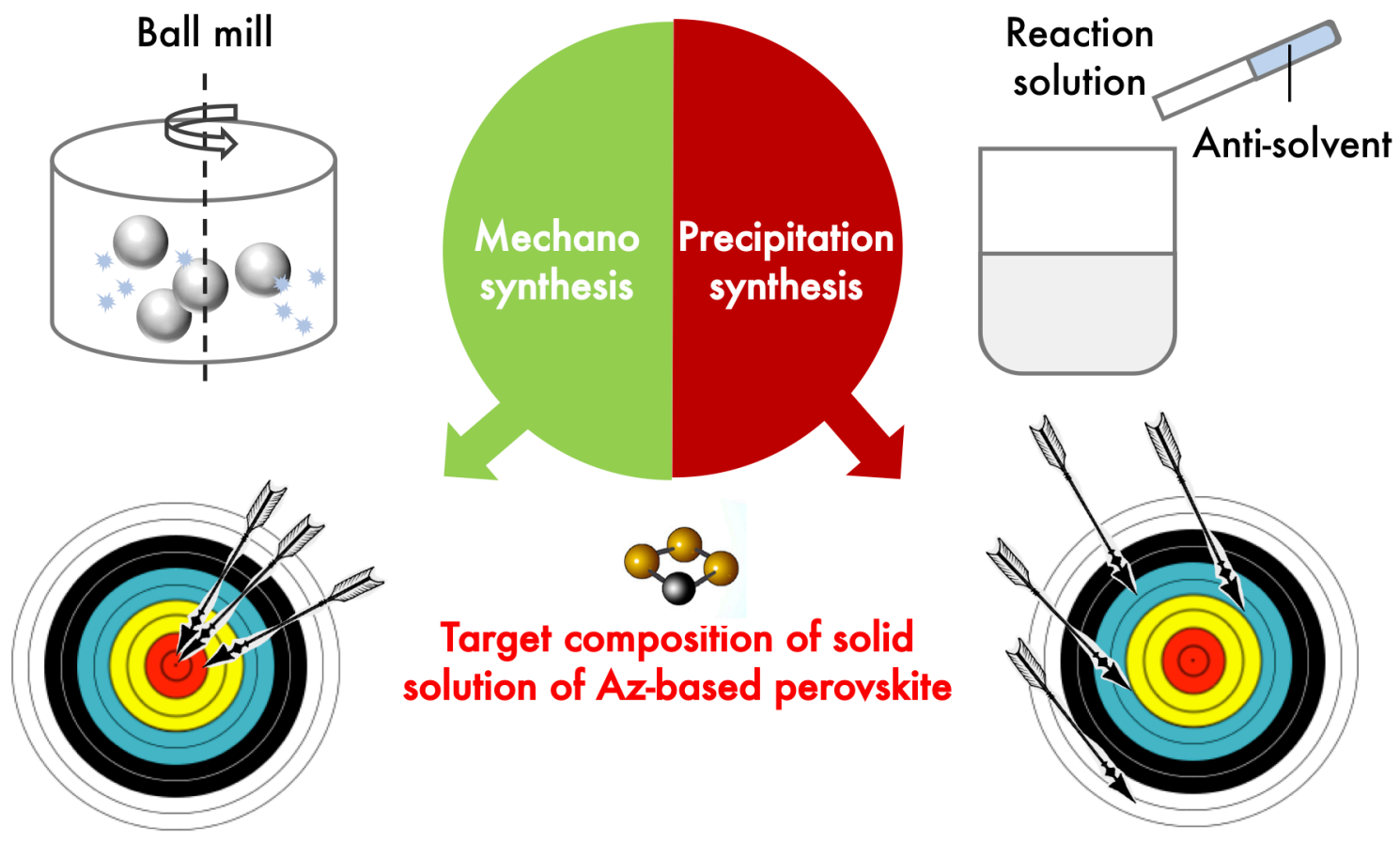

Target composition of solid solution of Az-based perovskite

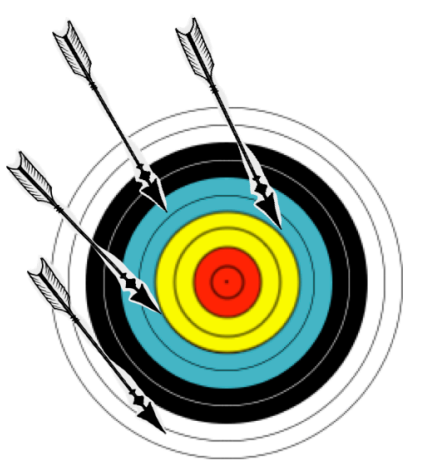

